\title{
Z3 $\begin{array}{ll}\text { Research Square } & \begin{array}{l}\text { They should not be considered conclusive, used to inform clinical practice, } \\ \text { or referenced by the media as validated information. }\end{array}\end{array}$
}

\section{Texture Analysis of The Microstructure of Internal Curing Concrete Based On Image Recognition Technology}

\section{Lixia Guo}

North China University of Water Resources and Electric Power

\section{Weikai Wang}

North China University of Water Resources and Electric Power

\section{Ling Zhong (D250514782@qq.com )}

North China University of Water Resources and Electric Power

\section{Lei Guo}

North China University of Water Resources and Electric Power

\section{Fangfang Zhang}

North China University of Water Resources and Electric Power

\section{Yuhang Guo}

North China University of Water Resources and Electric Power

\section{Research Article}

Keywords: internal curing concrete, scanning electron microscopy, Tamura texture, compressive strength, gray level co-occurrence matrix

Posted Date: November 30th, 2021

DOl: https://doi.org/10.21203/rs.3.rs-1085637/v1

License: (9) This work is licensed under a Creative Commons Attribution 4.0 International License.

Read Full License 


\title{
Texture analysis of the microstructure of internal curing concrete based on image recognition technology
}

\author{
Lixia Guo ${ }^{1,2,3}$ Weikai Wang ${ }^{1}$ Ling Zhong ${ }^{1,2,3 *}$ Lei Guo ${ }^{1,2,3}$ Fangfang Zhang ${ }^{1}$ \\ Yuhang Guo ${ }^{1}$
}

1 School of Water Conservancy, North China University of Water Resources and Electric Power,Zhengzhou 450046 ,China

2 Henan Water Valley Research Institute, Zhengzhou 450046, China

3 Henan Key Laboratory of Water Environment Simulation and reatment,Zhengzhou 450002, China

*Corresponding author, E-mail: 250514782@qq.com (Ling Zhong).

\begin{abstract}
Mechanical properties of internal curing concrete are greatly affected by its physical properties such as water content, cementing material content, porosity, and saturation. At the micro level, such impact is finally reflected in the surface texture of its materials. In this study, the image recognition technology was used to find that the internal curing concrete samples have significant micromorphology and texture features. A texture parameter-strength model was established based on the relationship between Tamura texture parameters, gray level co-occurrence matrix (GLCM) texture parameters, and the mechanical strength. Due to the characteristics of materials and the sensitivity of parameters, not all Tamura and GLCM texture parameters can effectively characterize the texture features of internal curing concrete materials. In terms of the Tamura texture, coarseness, regularity, and directionality are effective parameters to predict the compressive strength of the internal curing concrete. In terms of the GLCM texture, energy, correlation, entropy, and contrast are effective parameters to predict the compressive strength of the internal curing concrete. Correlations between each texture parameter and compressive strength follow different laws.
\end{abstract}

Keywords: internal curing concrete; scanning electron microscopy; Tamura texture; compressive strength; gray level co-occurrence matrix

\section{Introduction}

With the increasing demand for concrete in engineering construction, the superiority of high-strength concrete in water conservancy, bridges, ports and other projects have become increasingly prominent [1]. Compared with normal concrete, high-strength concrete features a lower water-binder ratio and a higher cement content, and also enjoys higher strength, impermeability, and durability, since it contains some ultrafine mineral admixtures such as silica fume and fly ash [2]. Previous studies about the properties of concrete materials are mostly made based on macro-mechanical experiments [3]; however, the macro-properties of materials are determined by their microstructures, and the changes in micromorphology are ultimately reflected in the texture features of the materials. Therefore, the study of the micro-properties of materials can allow us better understand their macro-deformation and failure mechanisms under different stress-strain conditions [4].

In recent years, modern detecting instruments have provided more possibilities for increasingly complex test conditions. The digital image-based modern detection methods have matured, and computer processing and image analysis have been widely used in various fields of science and technology [5,6]. At present, most researchers have analyzed the pore structure of cement-based 
materials using the image processing technology; however, in these studies, an abundance of information contained in images was not used effectively, except the gray level. Zhao Yan [7] et al. used ImageJ software to automatically extract solid particles from mudslides and found that this method can effectively retain the particles' size and roundness. Liu [8] et al. developed a pore (particle) and crack analysis system (PCAS). Through this system, geometrical and statistical parameters of particles and pores can be obtained. Yan Gaoyuan [9] et al. used a pore feature analysis system to extract pores in Ar-SEM images for quantitative characterization. Results showed that the pores are dominated by organic pores, intergranular pores, and intragranular pores. Zhou Shuangxi [10] et al. elaborated on the rapid analysis method for concrete pores based on deep learning. This method has good operability and typicality, and can improve analysis efficiency, suitable for in-depth studies of the correlation between concrete pore structure and macro-performance. Generally, pre-processing such as contrast enhancement is needed before the analysis of concrete pore structures in an image. For example, Zhang Xiong [11] et al. first blackened the cut and polished concrete cross-section with a marker, and then filled the pores with nano- $\mathrm{CaCO}_{3}$ to increase the contrast. Using the image processing technology, Qi Xuebin [12] et al. extracted the total pore area, fractal dimension, weighted probability entropy, and other parameters to characterize the geometrical and morphological characteristics of the pores, and through the micro-parameters to characterize the macro-mechanical properties of the materials. Each material has its own texture features. At present, image-based texture analysis has been widely used in various fields $[13,14]$. Since wood is a typical texture-type object, there have been many studies on its texture features. Zhao Peng [15] et al. proposed a wood species classification method based on I-BGLAM texture features. They compared it with conventional texture feature recognition methods and found that this method increases the accuracy of wood species classification. Hu Zhongkang [16] et al. proposed a texture segmentation method based on the gray level co-occurrence matrix (GLCM), which can effectively segment the wood surface defect images. Stefania [17] et al. integrated the eigenvector of the local binary pattern and the GLCM-based method to provide information about the image mode and volume data such as contrast and uniformity. Cement-based materials also have significant texture features [18], and changes in their pore structure may result in different textures and texture features. However, there are few experiments and studies on the texture features of cement-based materials. The texture feature extraction method provides a feasible idea for the research on the cement-based materials' performance.

This paper explores the micromorphology and texture features of internal curing concrete. Different texture feature extraction methods were used to analyze the test results. Tamura texture and GLCM were used to extract texture parameters. These parameters were analyzed to capture their correlations with the material's macro-mechanical properties. A model between the texture parameters and mechanical properties of internal curing concrete materials was established, providing a basis for theoretical research on the relationship between the material's microstructure and macro-mechanical properties.

\section{Materials and methods}

\subsection{Raw materials and test design}

The cement used was $\mathrm{P} \bullet \mathrm{O} 42.5$ Portland cement produced by Henan Tianrui Co., Ltd. The fine aggregates were collected from the Yaohe sand production area, with a particle size of $0.35-4.75 \mathrm{~mm}$ (medium sand). The coarse aggregates used 5-20 mm crushed limestone with continuous grading (Table 1). The slag, silica fume, and fly ash all met the requirements of GB/T18046-2017 and 
GB/T1596-2017 on admixtures. The main chemical composition of cementing materials is shown in Table 2. The mixing water used the laboratory's tap water. The water reducing agent used polycarboxylate superplasticizer. The super absorbent polymer (SAP) was purchased from Gongyi Best Green Materials Co., Ltd., with a specification of 60-100 meshes (particle size of $0.01-0.25 \mathrm{~mm}$ ) and a water absorbency of $400-500 \mathrm{~g} / \mathrm{g}$. The concrete was mixed using the pre-mixed cement paste method where the cementing materials were mixed with water to form cement paste and then mixed with aggregates and pre-absorbed SAP.

$150 \times 150 \times 150 \mathrm{~mm}$ standard concrete cubes with a water-cement ratio of 0.34 were prepared and cured for 28 days before the compressive strength test. The mix proportion is shown in Table 3. Assuming that the amount of water required for internal curing is equal to the amount of water released by the internal curing material, the content of the internal curing material is calculated according to the formula (1):

$$
V_{W}=\frac{C_{f} \times C S \times \alpha_{\max }}{\rho_{W}}
$$

Where $V_{W}$ is the maximum water diversion, $\mathrm{m}^{3} ; \rho_{W}$ is the density of water, $1,000 \mathrm{~kg} / \mathrm{m}^{3}$; $C_{f}$ is the amount of cement per unit volume of concrete, $\mathrm{m}^{3} ; C S$ is the chemical shrinkage ratio of cement after the complete hydration, which was adjusted to the chemical shrinkage ratio of cementing materials after the complete hydration in this study, $0.064 \mathrm{~mL} / \mathrm{g} ; \alpha_{\max }$ is the maximum degree of hydration. When the water-cement ratio is greater than or equal to 0.36 , the cement is considered fully hydrated, and $\alpha_{\max }=1$; when the water-cement ratio is less than $0.36, \alpha_{\max }=$ the water-cement ratio/0.36

Upon calculation, the cementing material content was $531.7 \mathrm{~kg}$, the SAP content was $0.12 \%$ and $0.24 \%$ of the cementing material content, and the pre-water absorption was 25 times the mass of SAP. The mixing amount of SAP was calculated at 25 times its water absorption rate.

Table 1 Physical properties of coarse aggregate

\begin{tabular}{cccccc}
\hline Project & $\begin{array}{c}\text { Apparent Density } \\
\left(\mathrm{kg} / \mathrm{m}^{3}\right)\end{array}$ & $\begin{array}{c}\text { Bulk Density } \\
\left(\mathrm{kg} / \mathrm{m}^{3}\right)\end{array}$ & $\begin{array}{c}\text { Porosity } \\
(\%)\end{array}$ & $\begin{array}{c}\text { Mud Content } \\
(\%)\end{array}$ & $\begin{array}{c}\text { Water } \\
\text { Absorption }(\%)\end{array}$ \\
\hline Standard & $>2500$ & $>1350$ & $<47$ & $<0.5$ & - \\
Measured & 2640 & 1720 & 40 & - & 1.2 \\
\hline
\end{tabular}

Table 2 Main chemical composition of cementitious materials ( \%)

\begin{tabular}{ccccccccc}
\hline Materials & $\mathrm{SO}_{3}$ & $\mathrm{SiO}_{2}$ & $\mathrm{Fe}_{2} \mathrm{O}_{3}$ & $\mathrm{Al}_{2} \mathrm{O}_{3}$ & $\mathrm{CaO}$ & $\mathrm{MgO}$ & $\mathrm{K}_{2} \mathrm{O}$ & $\mathrm{Na}_{2} \mathrm{O}$ \\
\hline Fly ash & 0.42 & 50.25 & 5.35 & 34.2 & 4.5 & 0.5 & 1.2 & 0.8 \\
$\begin{array}{c}\text { Silica Fume } \\
\text { Slag }\end{array}$ & & 90.48 & 1.87 & 1.27 & 2.59 & 0.91 & & \\
\hline
\end{tabular}


Table 3 Mix proportion of concrete (unit: $\mathrm{kg} / \mathrm{m}^{3}$ )

\begin{tabular}{|c|c|c|c|c|c|c|c|c|c|c|c|}
\hline Number & $\begin{array}{l}\text { Water } \\
\text { Cement } \\
\text { Ratio }\end{array}$ & Cement & Slag & FA & $\begin{array}{l}\text { Silica } \\
\text { Fume }\end{array}$ & Water & Sand & $\begin{array}{c}\text { Coarse } \\
\text { Aggregate }\end{array}$ & $\begin{array}{l}\text { Internal } \\
\text { Curing } \\
\text { Materials }\end{array}$ & $\begin{array}{c}\text { Water } \\
\text { Quantity } \\
\text { in } \\
\text { Advance }\end{array}$ & $\begin{array}{c}\text { Water } \\
\text { Reducing } \\
\text { Agent }\end{array}$ \\
\hline $\mathrm{HSC}$ & 0.34 & 371.2 & 79.6 & 53 & 26.4 & 180 & 625 & 1065 & 0 & 0 & 0.53 \\
\hline HSC5 & 0.34 & 371.2 & 79.6 & 53 & 26.4 & 180 & 625 & 1065 & 0.638 & 16 & 0.53 \\
\hline HSC10 & 0.34 & 371.2 & 79.6 & 53 & 26.4 & 180 & 625 & 1065 & 1.276 & 32 & 0.53 \\
\hline
\end{tabular}

Note: HSC refers to high-strength concrete. HSC5: SAP content is $0.12 \%$ of the cementing materials; HSC10: SAP content is $0.24 \%$ of the cementing materials.

\subsection{Experimental methods}

\subsubsection{Mechanical property test}

The compression test was carried out in accordance with the GB/T50081-2019 Standard for Test Methods of Concrete Physical and Mechanical Properties. The test data was also processed in line with this Standard.

$150 \times 150 \times 150 \mathrm{~mm}$ reference blocks were prepared for tests. Concrete blocks were taken out of the curing room and wiped. Then, they were put flat in the center of the lower platen of the press, with the molding surface facing the upper platen. A WAW-1000 electro-hydraulic servo universal testing machine was used in this test, and the loading speed was $8 \mathrm{KN} / \mathrm{S}$. Data meeting the requirements were retained, and data failing to meet the requirements were removed. Repeated tests were performed.

\subsubsection{Scanning electron microscopy (SEM)}

The FEI Quanta 650FEG SEM-EDS system was adopted to obtain the electron micrographs of the samples. To ensure the rationality of the test results, the samples used in the SEM test were randomly taken from the fragments crushed in the compression test. The samples were about $1 \mathrm{~cm}^{3}$ in size and retained their original forms, but the surface was flat through observation. A few fragments were tweezered with their fracture surface upwards and fixed on the conductive adhesive of the sample pedestal. Before the test, the hydration was stopped with ethanol for 24 hours. All samples were extracted from the ethanol solution and placed in a vacuum drying oven at $40^{\circ} \mathrm{C}$ for later use. The samples were sprayed gold with a coating thickness of $60 \sim 80 \mu \mathrm{m}$. SEM micrographs were obtained through FEI Quanta650FEG at 25 30 kV. The maximum current was $200 \mathrm{nA}$, the working distance was $10 \mathrm{~mm}$, and the dwell time was about 150 seconds. This test was carried out under the same conditions and in a stable environment to avoid errors during the test process. The samples were amplified by 1,000 to 5,000 times.

\subsection{Texture feature extraction methods}

\subsubsection{GLCM}

GLCM is an image processing method used to describe the spatial relationship of pixels in an image $[19,20]$, and it is established based on changes in the gray level from a reference pixel to its neighboring pixels. In texture recognition and segmentation, the texture measurement based on 
second-order statistics is superior to other methods. Therefore, this work also selects this method and another four typical texture features, namely energy, entropy, contrast, and correlation to extract eigenvalues [21].

In an $M \times N$ image, the GLCM element $P$ corresponds to the relative occurrence frequency of gray levels $i$ and $j$ of a pixel pair separated spatially. There is a point $i(x, y)$ and a point $j(x+d x, y+d y)$ in the image. The specific distance between the two points is expressed by $\delta$. The distance between the two points and the coordinate axes is expressed by $\theta$. The formula is as follows:

$$
p(i, j, \delta, \theta)=\{(x, y),(x+d x, y+d y) \in M \times N \mid f(x, y)=i, f(x+d x, y+d y)=j\}
$$

(1) Energy (angular second moment)

$$
\operatorname{energy}(A S E)=\sum_{i=1}^{g} \sum_{j=1}^{g} p^{2}(i, j, \delta, \theta)
$$

Where $p$ is the occurrence frequency of each gray-level pair, $\theta$ is the direction, and $g$ is the gray level.

Energy, also called angular second moment, is an indicator that measure the gray-level distribution and texture fineness of an image. The finer the texture is, the larger the energy is; the less uniform the gray-level distribution is, the smaller the energy is.

(2) Correlation

$$
\text { correlation }=\sum_{i=1}^{g} \sum_{j=1}^{g}\left[i \times j \times p(i, j, \delta, \theta)-u_{1} \times u_{2}\right] /\left(\delta_{\mathrm{x}} \times \delta_{y}\right)
$$

Where $u_{1}=\sum_{i=1}^{g} i \sum_{j=1}^{g} p(i, j, \delta, \theta), u_{2}=\sum_{i=1}^{g} j \sum_{j=1}^{g} p(i, j, \delta, \theta)$,

$\delta_{x}=\sum_{i=1}^{g}\left(i-u_{1}\right)^{2} \sum_{j=1}^{g} p(i, j, \delta, \theta)$

Correlation is used to measure the similarity of spatial GLCM elements in a row or column. When the element values are uniformly equal, the correlation is significant, and vice versa. If an image has a horizontal texture, the GLCM correlation value in the $0^{\circ}$ direction is usually greater than that in other directions. Therefore, it can be used to determine the main direction of the texture.

(3) Entropy

$$
\text { entropy }=\sum_{i=1}^{g} \sum_{j=1}^{g} p(i, j, \delta, \theta)^{2} \times \log _{10} P(i, j, \delta, \theta)
$$

Entropy is used to measure the amount of information in an image and indicates the complexity of the texture. If an image is highly complex, the entropy and fractal dimension will become large. If an image is less complex, the entropy and fractal dimension will become small.

(4) Contrast

$$
\text { contrast }=\sum_{i=1}^{g} \sum_{j=1}^{g}\left[(i-j)^{2} \times P^{2}(i, j, \delta, \theta)\right]
$$


Contrast reflects the sharpness of an image and the groove depth of its texture. The deeper the grooves are, the larger the contrast is, and the clearer the image is; on the contrary, the shallower the grooves are, the smaller the contrast is, and the vaguer the image is. The above four statistical parameters can be used together as characteristic parameters for texture analysis.

\subsubsection{Tamura texture}

TAMURA [22] et al. proposed the Tamura texture based on the psychology of visual perception. Tamura texture features correspond to the attributes of psychological texture, namely coarseness, contrast, directionality, linearity, regularity, and roughness.

(1) Coarseness

a) For each pixel $(x, y)$, calculate the average value of pixels in the $2^{\mathrm{k}} \times 2^{\mathrm{k}}$ neighborhood with the pixel at the center, as shown in the formula (7):

$$
A_{\mathrm{k}}(x, y)=\frac{1}{2^{2 \mathrm{k}}} \sum_{i=x-2^{k-1}}^{x+2^{k-1}} \sum_{j=y-2^{k-1}}^{y+2^{k-1}} f(i, j)
$$

Where $k=0,1,2,3,4,5$, and $f(i, j)$ is the gray level of the pixel at the point $(i, j)$.

b) For each pixel $(x, y)$, calculate the average intensity difference of non-overlapping windows in the horizontal direction, as shown in the formula (8), and also calculate the average intensity difference of non-overlapping windows in the vertical direction, as shown in the formula (9):

$$
\begin{aligned}
& E_{\mathrm{k}, \mathrm{h}}(x, y)=\left|A_{k}\left(x-2^{k-1}, y\right)-A_{k}\left(x+2^{k-1}, y\right)\right| \\
& E_{\mathrm{k}, v}(x, y)=\left|A_{k}\left(x, y-2^{k-1}\right)-A_{k}\left(x, y+2^{k-1}\right)\right|
\end{aligned}
$$

For each pixel $(x, y), E(x, y)=\max \left\{E_{k, h}(x, y), E_{k, v}(x, y)\right\}, \mathrm{k}=0,1, \ldots, 5$, the $k$ value corresponding to $E(x, y)$ is used to design the optimal size of the pixel $(x, y), S_{b e s t}(x, y)=2^{k}$.

c) With the pixel $(x, y)$ at the center, calculate the average coarseness in the $S_{\text {best }}(x, y) \times S_{\text {best }}(x, y)$ neighborhood, as shown in the formula (10):

$$
E_{k}=E_{\max }=\max \left(E_{1 h}, E_{1 v}, \ldots, E_{k h}, E_{k v}\right)
$$

d) Calculate the coarseness

$$
\begin{gathered}
S_{b e s t}(x, y)=2^{E_{\mathrm{k}}} \\
F_{\text {crs }}=\frac{1}{m \times n} \sum_{i=1}^{m} \sum_{j=1}^{n} S_{b e s t}(x, y)
\end{gathered}
$$

(2) Contrast

Contrast refers to the difference between the gray levels of the brightest part and the darkest part in a selected area of an image, and it can be obtained by statistical analysis of the gray level distribution around the pixels. Its calculation formula is as follows: 


$$
F_{\text {con }}=\sum_{n} n^{2}\left(\sum_{|i-j|=n} \sum_{|i-j|=n} p(i, j)\right)
$$

Where $p(i, j)$ is the $(i, j)$-th item in the $n \times n$ spatial gray-level independence matrix.

(3) Directionality

a) Calculate the gradient of each pixel, including the horizontal gradient $\Delta_{H}$ and the vertical gradient $\Delta_{V}$, which respectively represent the horizontal and vertical differences between the measured pixels. Convolute the image based on the formula (14). The horizontal gradient is equal to the sum of three gray level differences between the left and right pixels, and the vertical gradient is the deviation between the upper and lower pixels, as shown in the formula (15).

$$
\begin{gathered}
{\left[\begin{array}{lll}
-1 & 0 & 1 \\
-1 & 0 & 1 \\
-1 & 0 & 1
\end{array}\right]\left[\begin{array}{ccc}
1 & 1 & 1 \\
0 & 0 & 0 \\
-1 & -1 & -1
\end{array}\right]} \\
\Delta_{H}=\sum_{k \in(-1,0,1)}[p(i+1, j+k)-p(i-1, j+k)] \\
\Delta_{V}=\sum_{k \in(-1,0,1)}[p(i+k, j+1)-p(i+k, j-1)]
\end{gathered}
$$

b) Calculate the gradient vector

$$
\begin{aligned}
& |\Delta G|=\frac{\left|\Delta_{H}\right|+\left|\Delta_{V}\right|}{2} \\
& \phi=\tan ^{-1}\left(\frac{\Delta_{H}}{\Delta_{v}}\right)+\frac{\pi}{2}
\end{aligned}
$$

Where $\phi$ value is a real number $(0 \leq \phi \leq \pi)$, measured counterclockwise.

c) Calculate the histogram $H_{D}(k)$

$$
H_{D}(k)=N_{\phi}(k) / \sum_{i=0}^{n-1} N_{\phi}(i), k=0, \ldots, n-1
$$

Where $N_{\phi}(k)$ is the ratio of pixels where $\frac{2 k-1}{2 n}<\frac{\phi}{\pi}<\frac{2 k+1}{2 n}$ and $|\Delta G|>t$ are located. To prevent counting in unreliable directions, $|\Delta G|$ is determined by the value of $t$. Previous studies showed that the shape of $H_{D}(k)$ is not sensitive to the value of $t$, so this study took $t=12, n=16$ [23].

d) Calculate the directionality of the image by summing the variations around the peak. See literature [24]for details.

$$
F_{d i r}=1-r \cdot n_{p} \cdot \sum_{p}^{n_{p}} \sum_{\phi \in w_{p}}\left(\phi-\phi_{p}\right)^{2} H_{D}(\phi)
$$

(4) Regularity

The regularity can be calculated by the following formula, 


$$
F_{\mathrm{reg}}=1-r\left(\sigma_{c r s}+\sigma_{c o n}+\sigma_{d i r}+\sigma_{\text {lin }}\right)
$$

Where $r$ is a normalization factor, and $\sigma_{x}$ represents the standard deviation of $F_{x}$.

\section{Results and discussion}

SEM images with the magnification of 1,000 and 5,000 were selected, as shown in Figures 1 (1)(3). All selected images were from the same batch of samples and had the same size. They were used in the same environment and under the same test conditions. It can be seen from these figures that the material itself has obvious texture features. As the SAP content changes, the concrete morphology and mechanical properties change accordingly. In this study, Tamura texture and GLCM were used to calculate the SEM image characteristic parameters, and relations between these parameters and compressive strength were established.

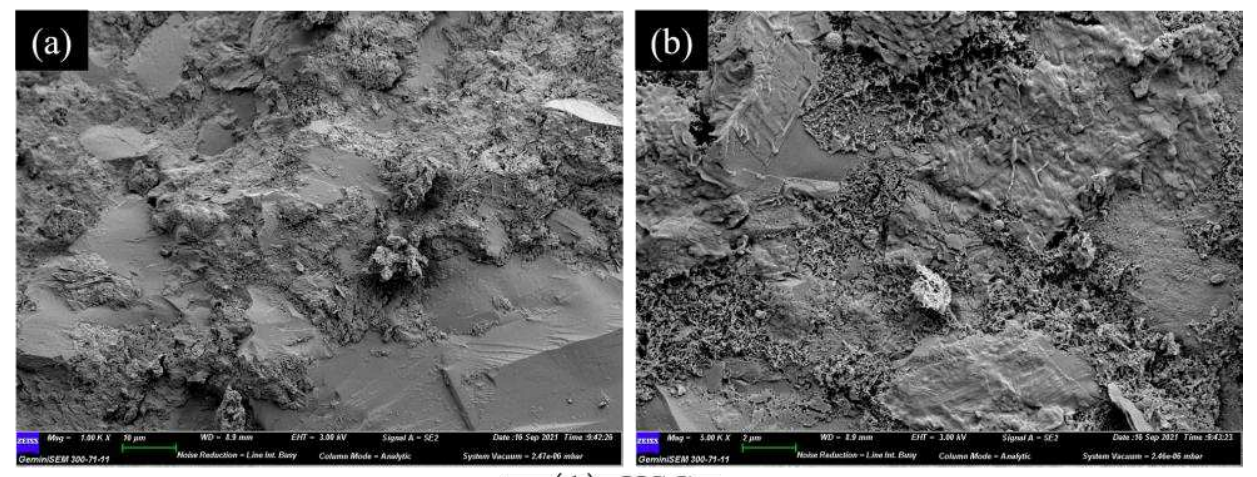

(1) HSC

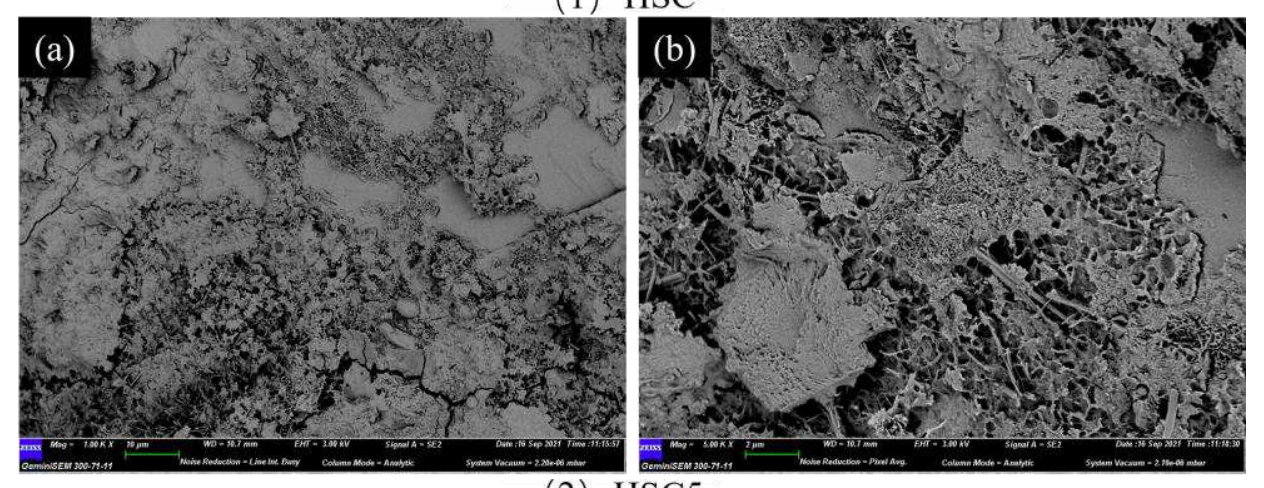

(2) HSC5

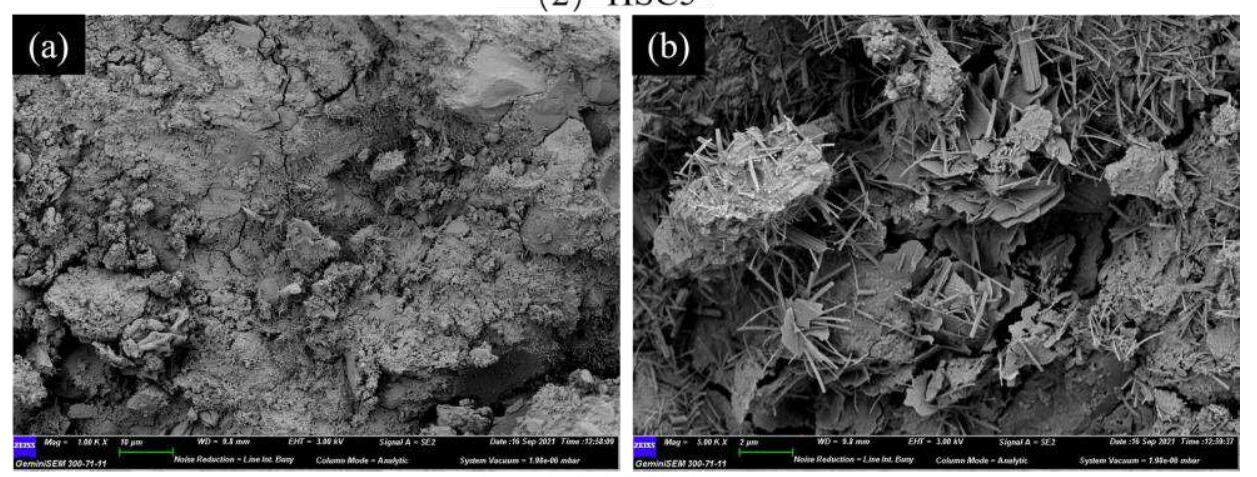

(3) $\mathrm{HSC10}$

Fig. 1 SEM images of specimens with different SAP contents (1000× and 5000× magnification): (1) HSC specimen; (2) HSC5 specimen; (3) HSC10 specimen

\subsection{GLCM characteristic parameters}


A self-compiled program was used to extract characteristic parameters from the SEM images. When extracting, the gray level (g) was 16, the distance (step length, d) between pixels was 1, and the direction $(\theta)$ was $0^{\circ}, 45^{\circ}, 90^{\circ}$, and $135^{\circ}$. The calculation results of these four characteristic parameters are shown in Table 4.

Table 4 GLCM characteristic value

\begin{tabular}{cccccc}
\hline Number & Energy & Entropy & Contrast & Correlation & Magnification \\
\hline HSC & 0.0223 & 4.1287 & 3.3307 & 0.1204 & \\
HSC5 & 0.0405 & 3.5243 & 1.0438 & 0.1804 & $1000 \times$ \\
HSC10 & 0.0712 & 2.8859 & 0.6229 & 0.3184 & \\
\hline HSC & 0.0287 & 3.8825 & 1.8677 & 0.1422 & \\
HSC5 & 0.0569 & 3.4109 & 1.7337 & 0.2212 & $5000 \times$ \\
HSC10 & 0.0728 & 3.06 & 1.5101 & 0.3071 & \\
\hline
\end{tabular}

As shown in Figure 2, the energy and correlation rise while the entropy and contrast fall with the increase of the SAP content. Whether the SEM image is amplified by 1,000 or 5,000 times, the GLCM characteristic parameters show a significant correlation with the SAP content. Upon comparison, the correlation is better when the SEM image is amplified by 1,000 times; therefore, this study selects $1,000 \times$ amplified images as samples for quantitative analysis [25].
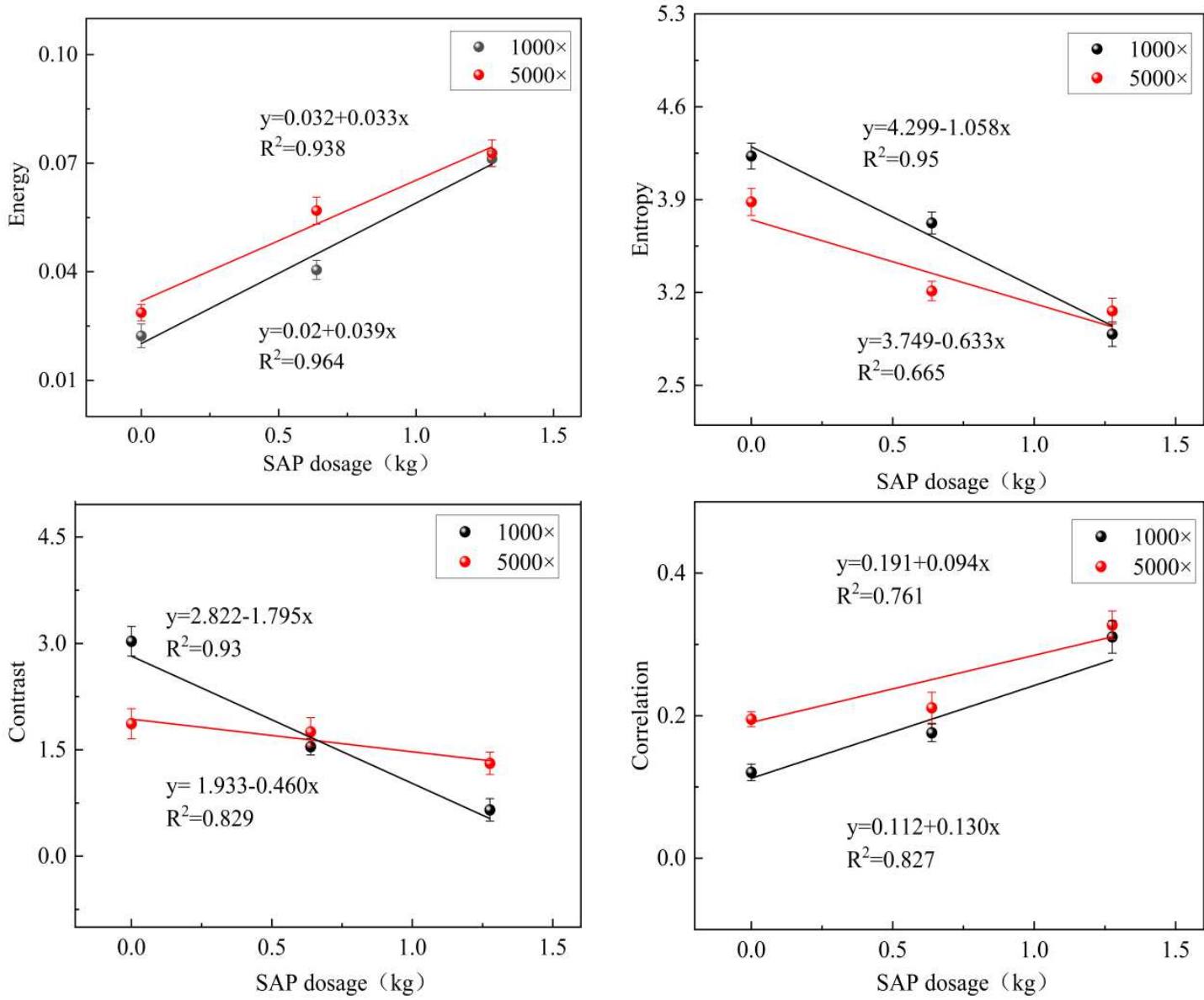

Fig. 2 GLCM characteristic values under different SAP internal curing materials 


\subsection{Tamura texture parameters}

Features of Tamura texture were captured on MATLAB software. A computing program was compiled to calculate the four features of Tamura texture, namely coarseness, contrast, directionality, and regularity. Table 5 lists the calculation results of the test samples.

Table 5 Tamura texture characteristic value

\begin{tabular}{cccccc}
\hline Number & Coarseness & Directionality & Contrast & Regularity & Magnification \\
\hline HSC & 17.2817 & 31.325 & 30.3632 & 0.9439 & \\
HSC5 & 17.4007 & 34.509 & 32.5402 & 0.9388 & $1000 \times$ \\
HSC10 & 17.6087 & 38.677 & 35.8368 & 0.9304 & \\
\hline HSC & 17.294 & 32.156 & 50.9525 & 0.9329 & \\
HSC5 & 17.5407 & 37.285 & 45.5003 & 0.9326 & $5000 \times$ \\
HSC10 & 18.0722 & 41.236 & 39.1313 & 0.9322 & \\
\hline
\end{tabular}

It can be seen from Figure 3 that the coarseness and directionality increase while the entropy and contrast decrease with the increase of the SAP content. Except for contrast, the correlation coefficient $\left(\mathrm{R}^{2}\right)$ between other characteristic values and the SAP content are all greater than $96 \%$. The correlation coefficient $\left(\mathrm{R}^{2}\right)$ between the texture features and the SAP content on a 5,000× amplified image is less than that on a $1,000 \times$ amplified image. Apparently, using the $1,000 \times$ amplified image as the analysis object can improve the reliability of the quantitative analysis. Therefore, this study selected an image with a magnification of 1,000 as a sample for quantitative analysis.
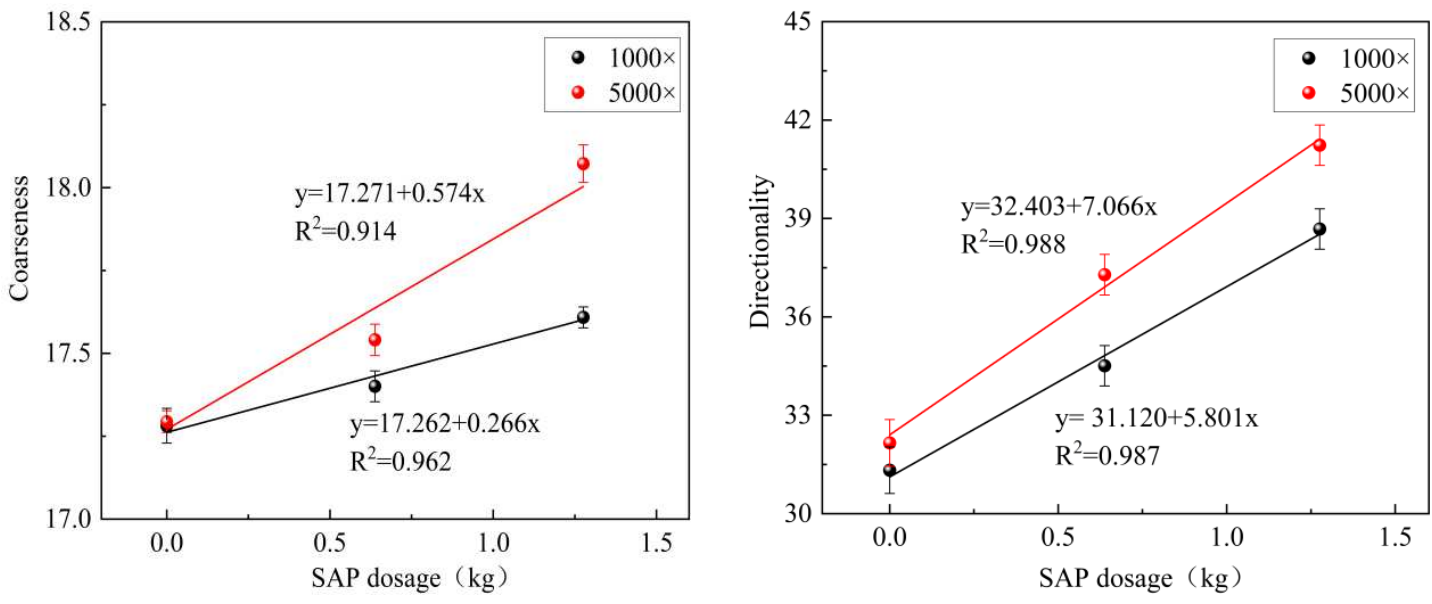

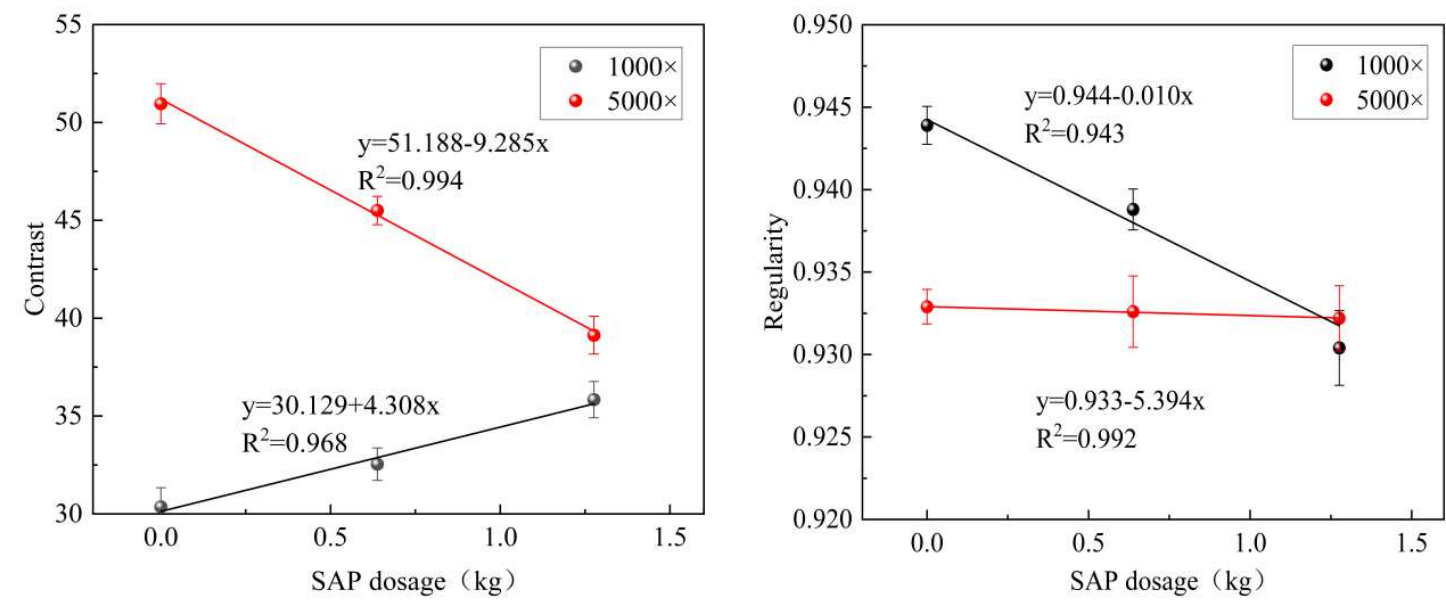

Fig.3 Tamura texture characteristic values under different SAP internal curing materials

\subsection{The relationship between texture features and compressive strength}

Based on the SEM images, the GLCM characteristic parameters and compressive strength of the internal curing concrete under different mix proportions were obtained, as shown in Figure 4. There is a significant correlation between the compressive strength and GLCM characteristic parameters (energy, contrast, correlation, entropy), and the correlation coefficients $\left(\mathrm{R}^{2}\right)$ are all higher than $90.4 \%$. Similar to Tamura texture features, each correlation follows different laws. For example, the compressive strength has a significant negative linear relation with the energy and correlation, and a significant positive linear relation with the contrast and entropy.
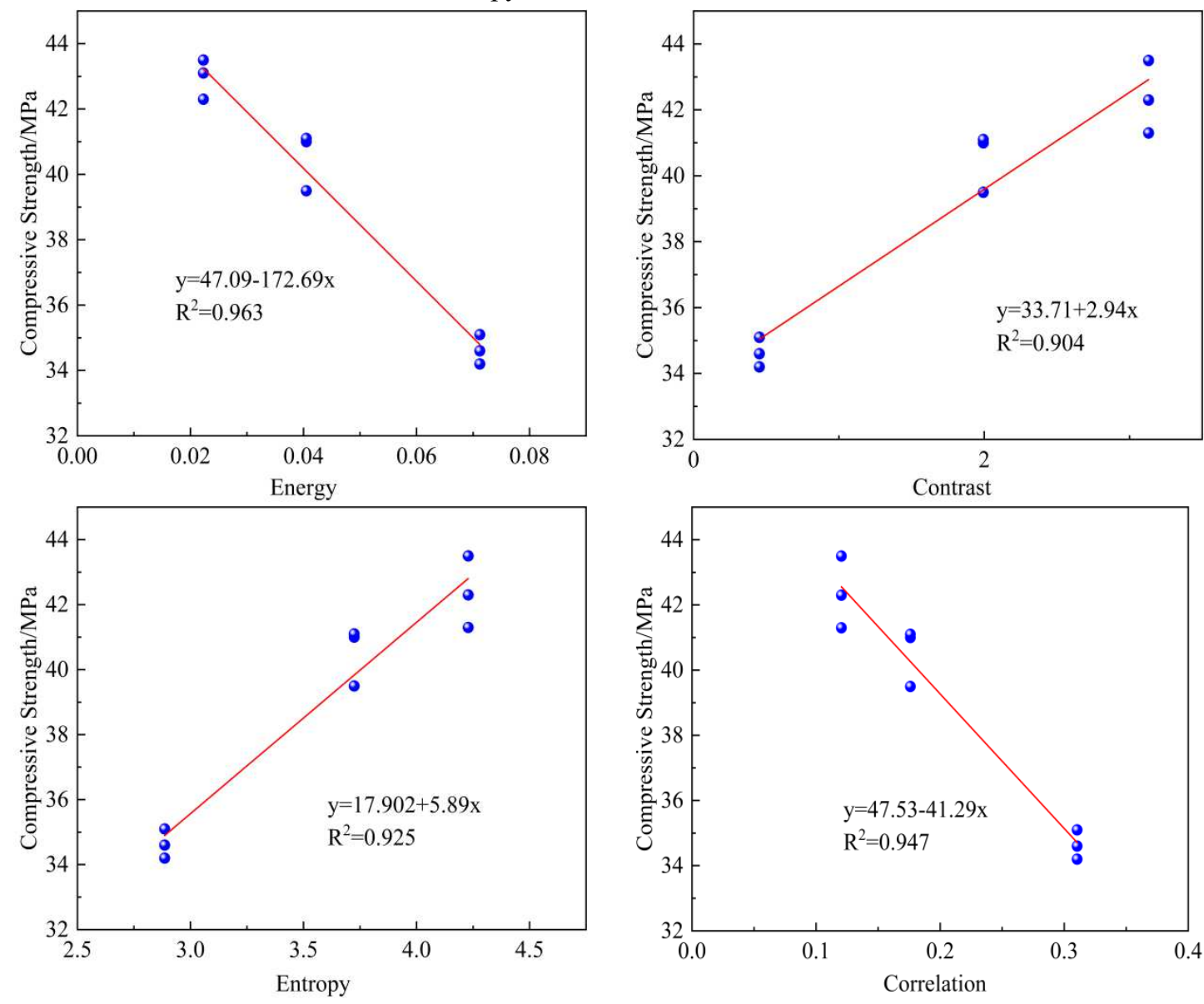

Fig.4 Relationship between strength and GLCM characteristic values 
To illustrate the relationship between the Tamura texture features and the compressive strength of the internal curing concrete samples based on the SEM images [26], the Tamura texture features and compressive strength of the samples under different mix proportions were obtained, as shown in Figure 5. It can be seen from the figure that there is a negative correlation between the comprehensive strength and the Tamura texture features (coarseness, directionality, and contrast), and a positive correlation between the comprehensive strength and the regularity. The correlation coefficients $\left(\mathrm{R}^{2}\right)$ of the three eigenvalues are all greater than $92.5 \%$. A test model was established based on the comprehensive strength and three different parameters to effectively predict the compressive strength of materials.

Studies showed that with the increase of the compressive strength, the Tamura texture parameters coarseness and directionality decrease [27].According to the SEM images, it was found that many tiny pores may appear during the SAP release, so higher SAP content will increase the texture's coarseness, which is consistent with the above research results, indicating that the higher the coarseness and irregularity of the pore structure in the concrete material, the lower the comprehensive strength. It also indicated that the texture features of samples depend on their pore structures, and the texture parameters can fully characterize mechanical properties of the materials.
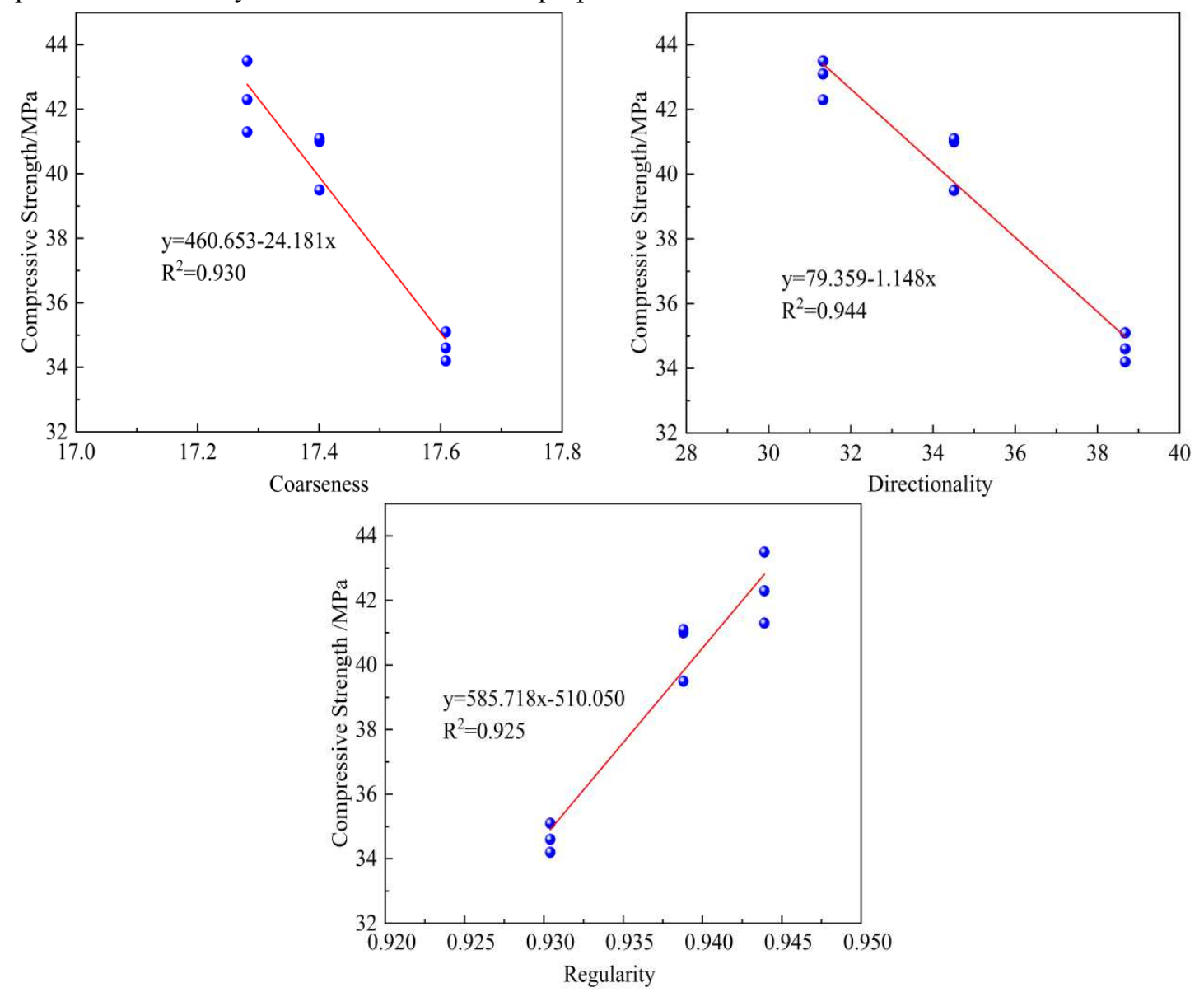

Fig.5 Relationship between strength and Tamura texture characteristic values

The mechanical properties of internal curing concrete materials are greatly affected by their physical properties such as water content, cementing material content, porosity, and saturation. At the micro level, the impact of the above indicators is reflected in the surface texture of the materials. Both Tamura and GLCM texture parameters can effectively quantify the morphological features of the concrete materials and further predict their mechanical strength. However, due to the characteristics of materials and the sensitivity of parameters, not all Tamura and GLCM texture parameters can 
effectively characterize the texture features of internal curing concrete materials. As shown in Figures 4 and 5, in terms of the Tamura texture, coarseness, regularity, and directionality are effective parameters to predict the compressive strength of the internal curing concrete materials. In terms of the GLCM texture, energy, correlation, entropy, and contrast are effective parameters to predict the compressive strength of the internal curing concrete materials.

\section{Conclusions}

This study proved that there is a correlation between Tamura texture parameters and mechanical strength, GLCM texture parameters and mechanical strength, and established a model based on the texture parameters and mechanical properties of internal curing concrete materials. The texture extraction methods and characteristic parameters suitable for internal curing concrete materials were selected for characterization. Based on the test results, the following conclusions were drawn:

(1) Proper texture feature extraction methods were used in this study to predict the macro-mechanical properties of the materials, providing new ideas for research on the macro-micro properties of the internal curing concrete.

(2) There is a significant correlation between compressive strength and GLCM characteristic parameters, and the correlation coefficients $\left(\mathrm{R}^{2}\right)$ are all higher than $90 \%$. Similar to Tamura texture features, each correlation follows different laws.

(3) In terms of the Tamura texture, coarseness, regularity, and directionality are effective parameters to predict the compressive strength of internal curing concrete. In terms of the GLCM texture, energy, correlation, entropy, and contrast are effective parameters to predict the compressive strength of $t$ internal curing concrete.

\section{Acknowledgments}

This work was funded by Natural Science Foundation of Henan Province: (202300410270) Research on Frost Resistance Durability Behavior and Deterioration Damage Mechanism of Cemented Sand and Gravel and National Natural Science Foundation of China: (5210090341) Macro and meso mechanism of damage evolution of cement-sand-gravel dam under extreme temperature.

\section{Data Availability}

The datasets generated during and analysed during the current study are available from the corresponding author on reasonable request.

\section{Reference}

[1] Akhnoukh, A. K. Internal curing of concrete using lightweight aggregates. Particul Sci Techno. 36(3), 362-367(2018)

[2] Zhan Binggen, Ding Yibing. Hydration of Cement Paste with Low Water-Cement Ratio

Incorporating Polyacrylate Super-absorbent Polymer[J].Journal of Building Materials, 2007(02):148-153.(in Chinese)

[3] Guo Lei,Wang Jia,Guo Lixia,et al. Effect of Mud Content in Cemented Sand Gravel Based on Its Strength[J]. Yellow River, 2020,42(03):136-139. (in Chinese)

[4] Liu Chun,Xu Qiang,Shi Bin,et al. Digital image recognition method of rock particle and pore system and ts application[J]. Chinese Journal of Geotechnical Engineering, 2018,40(05):925-931. (in Chinese)

[5] Asgharnejad, H., Sarrafzadeh, M. H., Abhar-Shegofteh, O., Nazloo, E. K., \& Oh, H. M. Biomass 
quantification and 3-D topography reconstruction of microalgal biofilms using digital image processing. Algal Res. 55, 102243(2021)

[6] Shi Liwan,Wang Duanyi,Wu Wenliang,et al.Study of Asphalt Mixture Skeleton Based on Digital Image Processing[J]. Journal of Building Materials, 2016,19(04):767-772. (in Chinese)

[7] Zhao Yan,Zheng Jiaoyu,Guo Peng,et al.Applications of the ImageJ software in analysis of solid grains in a debris flow gully[J]. Journal of Lanzhou University(Natural Sciences), 2015,51(06):877-881. (in Chinese)

[8] Liu, C., Shi, B., Zhou, J., \& Tang, C. Quantification and characterization of microporosity by image processing, geometric measurement and statistical methods: application on SEM images of clay materials. Appl Clay Sci. 54(1), 97-106(2011).

[9] Yan Gaoyuan,Wei Chongtao,Song Yu,et al.Quantitative Characterization of Shale Pore Structure Based on Ar-SEM and PCAS[J] Earth Science, 2018,43(05):1602-1610. (in Chinese)

[10] Zhou Shuangxi, Sheng Wei,Wei Xing,et al. Fast Image Analysis on Pore Structure of Concrete Based on Deep Learning[J]. Journal of the Chinese Ceramic Society, 2019,47(05):653-663. (in Chinese)

[11] Fang Yonghao,Wang Rui,Pang Erbo,et al. Relationship Between Compressive Strength and Air-void Structure Ofamed Cement-ash Concrete [J]. Journal of the Chinese Ceramic Society, 2010, 38(4): 621-626. (in Chinese)

[12] Qin, X., Wang, P., Liu, L., Wang, M., \& Xin, J. Sensitivity analysis of microstructure parameters and mechanical strength during consolidation of cemented paste backfill. Math Probl Eng. 2018(2018)

[13] Huang, C. L., Lian, M. J., Wu, Y. H., Chen, W. M., \& Chiu, W. T. Identification of Human Ovarian Adenocarcinoma Cells with Cisplatin-Resistance by Feature Extraction of Gray Level Co-Occurrence Matrix Using Optical Images. Diagnostics. 10(6), 389(2020)

[14] Song Yongchao,Liang Naixing,Yan Gongxi,et al. Skid-resistant performance of texture structure of exposed-aggregate cement concrete pavement based on digital image technology[J]. Journal of Harbin Institute of Technology, 2015,47(02):123-128. (in Chinese)

[15] Zhao Peng,Han Jincheng,Wang Chengkun,et al. Wood Species Classification With Microscopic Hyper-Spectral Imaging Based on I-BGLAM Texture and Spectral Fusion[J]. Spectroscopy and Spectral Analysis, 2021,41(02):599-605. (in Chinese)

[16] Hu Zhongkang,Liu Ying,Zhou Xiaolin,et al. Research on defects and textures recognition of solid wood lumbers based on deep belief network [J]. Application Research of Computers, 2019,36(12):3889-3892. (in Chinese)

[17] Barburiceanu, S., Terebes, R., \& Meza, S. 3D Texture Feature Extraction and Classification Using GLCM and LBP-Based Descriptors. Appl Sci. 11(5), 2332(2021)

[18] Cong Zhuohong,Chen Hengda,Zheng Nanxiang,et al. Surface Texture of Cement Concrete Pavement: a Review[J]. Materials Reports, 2020, 34(09):9110-9116. (in Chinese)

[19] Alvarenga, A. V., Teixeira, C. A., Ruano, M. G., \& Pereira, W. C. Influence of temperature variations on the entropy and correlation of the Grey-Level Co-occurrence Matrix from B-Mode images. Ultrasonics. 50(2), 290-293(2010)

[20] Honeycutt, C. E., \& Plotnick, R. Image analysis techniques and gray-level co-occurrence matrices (GLCM) for calculating bioturbation indices and characterizing biogenic sedimentary structures. Comput Geosci-UK. 34(11), 1461-1472(2008)

[21] Gómez, W., Pereira, W. C. A., \& Infantosi, A. F. C. Analysis of co-occurrence texture statistics as a function of gray-level quantization for classifying breast ultrasound. IEEE T Med Imaging. 31(10), 
1889-1899(2012)

[22] Amadasun, M., \& King, R. Textural features corresponding to textural properties. IEEE T Sys Man Cy. 19(5), 1264-1274 (1989).

[23] Zhao Haiying,Xu Guangmei,Peng Hong. Performance Evaluation for the Algorithms to Measure Texture Coarseness[J]. Computer Science, 2011,38(06):288-292. (in Chinese)

[24] Tamura, H., Mori, S., \& Yamawaki, T. Textural features corresponding to visual perception. IEEE T Syst Man Cy. 8(6), 460-473(1978)

[25] Guo, L., Zhang, F., Zhong, L., Guo, L., Wang, L., Wang, M., ... \& Li, S. Texture analysis of the microstructure of concrete with different concentrations of superabsorbent polymer after internal curing. Mater Today Commun. 27, 102361(2021)

[26] Yin, S. H., Shao, Y. J., Wu, A. X., Wang, Y. M., \& Gao, Z. Y. Texture features analysis on micro-structure of paste backfill based on image analysis technology. J Cent South Univ. 25(10), 2360-2372 (2018)

[27] Venkateswaran, K., Kasthuri, N., \& Alaguraja, R. A. A combined detail enhancing algorithm and texture feature extraction method for supervised classification of remote sensing images. J Indian Soc Remote. 46(3), 327-336(2018) 